\title{
Caffeine Overdose Complicated by Acute Kidney Injury despite Mild Creatine Kinase Elevation
}

\author{
Nao Koide ${ }^{a}$ Nobuhiro Sato ${ }^{a}$ Daisuke Kondo ${ }^{b}$ Yasuo Hirose ${ }^{a}$ \\ aDepartment of Emergency and Critical Care Medicine, Niigata City General Hospital, \\ Niigata City, Japan; ${ }^{b}$ Department of Nephrology, Niigata City General Hospital, Niigata \\ City, Japan
}

\section{Keywords}

Acute kidney injury · Caffeine $\cdot$ Drug overdose

\section{Abstract}

Caffeine is a commonly used stimulant in our society. Prior case reports have described acute caffeine overdose resulting in rhabdomyolysis and acute kidney injury (AKI). We present the case of a 29-year-old man who presented to the emergency department after ingesting $20.1 \mathrm{~g}$ of caffeine in a suicide attempt and experienced AKI with only mildly elevated creatine kinase (CK). This case highlights the possibility that AKI can result from a caffeine overdose, even if the patient's CK is only slightly elevated.

\section{Introduction}

Caffeine is a natural alkaloid that is obtained from coffee beans, tea leaves, cocoa beans, and kola nuts [1, 2]. It is included in drinks, prescription, and over-the-counter drugs, as well as weight loss supplements [2]. While caffeine is generally thought to be safe in moderate amounts, large doses produce clinical features including cardiovascular, neurological, or gastrointestinal symptoms [3]. 
Acute kidney injury (AKI) secondary to rhabdomyolysis due to caffeine overdose has been reported $[4,5]$. Herein, we describe a case of AKI with mildly elevated creatine kinase (CK) caused by a caffeine overdose.

\section{Case Report}

A previously healthy 29-year-old man presented to the urgent care center with nausea, vomiting, and mild disturbance of consciousness. He described taking 100 caffeine tablets and a small amount of a general cold drug that includes acetaminophen, $600 \mathrm{mg}$; guaifenesin, 120 $\mathrm{mg}$; caffeine, $50 \mathrm{mg}$; DL-methylephedrine hydrochloride, $40 \mathrm{mg}$; dihydrocodeine phosphate, $16 \mathrm{mg}$; riboflavin sodium phosphate, $8 \mathrm{mg}$; and d-chlorpheniramine maleate, $5 \mathrm{mg}$ (for a combined total of $20.1 \mathrm{~g}$ of caffeine) 1 day previously in a suicide attempt. He was transferred to our emergency department (ED).

The patient's vital signs on ED presentation included blood pressure, 124/65 mm Hg; heart rate, 99 beats/min; pulse oximetry, $100 \%$ on room air; and body temperature, $37.8^{\circ} \mathrm{C}$. There were no respiratory or abdominal findings. His Glasgow coma scale score was 14 . Electrocardiography revealed a normal sinus rhythm.

Laboratory findings showed the following: aspartate aminotransferase (AST), $42 \mathrm{U} / \mathrm{L}$; alanine aminotransferase (ALT), $17 \mathrm{U} / \mathrm{L}$; lactate dehydrogenase (LDH), $223 \mathrm{U} / \mathrm{L} ; \mathrm{CK}, 1,842 \mathrm{U} / \mathrm{L}$; total bilirubin, $0.5 \mathrm{mg} / \mathrm{dL}$; sodium, $144 \mathrm{mEq} / \mathrm{L}$; potassium, $3.5 \mathrm{mEq} / \mathrm{L}$; chloride, $97 \mathrm{mEq} / \mathrm{L}$; blood urea nitrogen, $26.4 \mathrm{mg} / \mathrm{dL}$; creatinine, $2.9 \mathrm{mg} / \mathrm{dL}$; lactate, $2.98 \mathrm{mmol} / \mathrm{L}$; white blood cell count, $18.1 \times 10^{9} / \mathrm{L}$ (neutrophils, $92.4 \%$; lymphocytes, 3.3\%; monocytes, $2.9 \%$; eosinophils, 0.8\%; and basophils, 0.6\%); hemoglobin, $14.7 \mathrm{~g} / \mathrm{L}$; and platelet count, $299 \times 10 \% / \mathrm{L}$. An arterial blood gas analysis showed mixed alkalosis $\left(\mathrm{pH}, 7.507 ; \mathrm{pCO}_{2}, 38.5 \mathrm{~mm} \mathrm{Hg}\right.$; $\mathrm{pO}_{2}, 65.2$ $\mathrm{mm} \mathrm{Hg} ; \mathrm{HCO}_{3}, 29.8 \mathrm{mEq} / \mathrm{L} ; \mathrm{BE}, 6.4 \mathrm{mEq} / \mathrm{L}$ ). His serum theophylline concentration was 8.95 $\mu \mathrm{g} / \mathrm{mL}$ and serum caffeine concentration was $35.2 \mathrm{mg} / \mathrm{L}$. A urine study revealed a $\mathrm{pH}$ of 6.5 ; proteinuria, 1+; ketonuria, 1+; hematuria, 3+; pyuria, 3+; red blood cells, 30-49/high-power field (HPF); white blood cells, 30-49/HPF; and urinary $\beta_{2}$-microglobulin, $14.93 \mathrm{mg} / \mathrm{L}$. The urine specific gravity was 1.011 , and the fractional excretion of sodium (FENa) was $2.52 \%$.

Although the patient received $1.5 \mathrm{~L}$ of crystalloid solution in the ED, he produced only 107 $\mathrm{mL}$ of urine in $6 \mathrm{~h}$. He remained hemodynamically stable and was admitted to the intensive care unit. The renal function did not show any improvements following an intravenous volume challenge, indicating that the prerenal AKI was unlikely. His highest CK concentration was 4,237 U/L on hospital day 3. Urinalysis showed red blood cells on light microscopy. His renal function gradually worsened, and his serum creatinine concentration was $5.49 \mathrm{mg} / \mathrm{dL}$ on hospital day 6 . There was no renal atrophy evident on echography or computed tomography. Renal scanning with ${ }^{67}$-gallium did not produce positive findings. His renal function gradually recovered without dialysis. He was discharged on hospital day 26. A renal biopsy was not performed. His creatinine concentration was $0.89 \mathrm{mg} / \mathrm{dL}$ at a follow-up visit to a nephrology clinic 1 month after hospital discharge.

\section{Discussion}

Caffeine is a ubiquitous drug in modern society and has dose-dependent effects. While intake levels below $400 \mathrm{mg}$ per day are generally thought to be safe in healthy adults, the fatal acute oral caffeine dose in adults is estimated to be in the range of $150-200 \mathrm{mg} / \mathrm{kg}$ body 
weight $[3,6]$. Alternatively, toxicological symptoms begin at concentrations of $15 \mathrm{mg} / \mathrm{L}$, and concentrations $\geq 80 \mathrm{mg} / \mathrm{L}$ are considered lethal [3]. Around 5\% of caffeine is metabolized to theophylline. The clinical features of caffeine intoxication include cardiovascular symptoms (hypertension, hypotension, dysrhythmia, myocardial ischemia, and cardiac arrest), neurological (delusions, hallucinations, anxiety, agitation, seizure, and coma), or gastrointestinal symptoms (nausea, vomiting, abdominal pain, and diarrhea) [3]. AKI secondary to rhabdomyolysis resulting from caffeine overdose has been reported [4,5]. A previous report described a 42-year-old man who ingested $24 \mathrm{~g}$ of caffeine. His CK concentration was 59,000 U/L 4 days after ingestion, and he required hemodialysis [5].

Rhabdomyolysis is characterized by the leakage of muscle-cell contents, including electrolytes, myoglobin, and other sarcoplasmic proteins (e.g., CK, LDH, AST, and ALT) into circulation $[7,8]$, and AKI is a potential complication of severe rhabdomyolysis. Although there is no defined $\mathrm{CK}$ value above which the risk of AKI markedly increases, some studies suggest that patients with CK levels $<5,000 \mathrm{IU} / \mathrm{L}$ are not at risk of developing AKI $[9,10]$. Alternatively, in a review, the risk of AKI in rhabdomyolysis is usually low when CK levels at admission are less than 15,000-20,000 U/L [8].

In our case, the patient experienced nausea, vomiting, mild disturbance of consciousness, and AKI after consuming $20.1 \mathrm{~g}$ of caffeine, even though his CK was only mildly elevated $(4,237$ $\mathrm{U} / \mathrm{L}$ ). This case did not conform to the usual testing standards that are relied upon for a diagnosis of rhabdomyolysis. An increasing number of studies have demonstrated that caffeine contributes to the development and exacerbation of various kidney diseases [11]. Therefore, the possibility of AKI secondary to something other than rhabdomyolysis must be considered. The case we present had a relatively low BUN-to-creatinine ratio; urine testing on admission (not concentrated, with a FENa of $2.52 \%$, urine specific gravity of 1.011 , hematuria, and pyuria), suggested that his AKI was not caused by volume depletion, such as dehydration $[12,13]$. AKI with intrinsic renal injury from three possible mechanisms is likely. The first possibility is acute interstitial nephritis (AIN). Drug-induced renal interstitial inflammation commonly manifests with a predominance of lymphocytes, monocytes, eosinophils, and plasma cells within the interstitium; active urinary sediment with pyuria or white blood cell casts; eosinophiluria; hematuria; and mild to moderate proteinuria, and ${ }^{67}$-gallium scintigraphy could be a useful tool to detect AIN [14]. Urinary studies in our case showed pyuria, hematuria, and mild proteinuria. Previous studies have reported 31-42\% false negative results of ${ }^{67}$-gallium scintigraphy [15]; hence, in this case, AIN can be considered despite negative scintigraphy results. Although acute interstitial nephritis can only be definitely diagnosed by renal biopsy [16], renal biopsy was not performed because renal function was gradually recovered without dialysis in this case.

Another possibility is rhabdomyolysis without elevated CK. A prior case report showed that CK alone might not be a sensitive marker for diagnosing rhabdomyolysis [17]. Despite confirmatory myoglobin casts on renal biopsy in that case, the patient's CK concentration was only $156 \mathrm{U} / \mathrm{L}$. Unfortunately, renal biopsy was not performed in our case. Although another case described that AKI secondary to rhabdomyolysis occurred in a 21-year-old man who ingested $3.57 \mathrm{~g}$ of caffeine as a result of a caffeine overdose, that patient's CK concentration was relatively low for rhabdomyolysis (measured CK 1,134 IU/L 5 days after ingestion) [4]. Renal biopsy was not performed in that case, either, so the exact mechanism was not known. However, that case could have had a mechanism similar to that in our case. We speculated that the possibility of rhabdomyolysis caused by AKI was low in our case, owing to the presence of red blood cells on urinalysis, and the scarcity of previous reports on rhabdomyolysis without elevated $\mathrm{CK}$. 
Koide et al.: Caffeine Overdose Complicated by AKI despite Mild CK Elevation

The other possibility is AKI induced by other drugs. The patient we present took a small amount of a general cold drug. Therapeutic dose of acetaminophen is a possible risk factor for AKI [18]. Previous cases reported that acetaminophen caused acute tubular necrosis and acetaminophen-induced renal failure became evident after hepatotoxicity in most cases $[18,19]$. However, our case did not manifest hepatotoxicity, and renal biopsy was not performed. In addition, although guaifenesin and ephedrine abuse can cause AKI with nephrolithiasis [20, 21], our case had no nephrolithiasis.

\section{Conclusion}

Herein, we described a rare case of AKI with mildly elevated CK induced by a caffeine overdose. Physicians should consider the possibility of AKI following caffeine overdose even if CK is only mildly elevated.

\section{Statement of Ethics}

Written informed consent was obtained from the case for publication of this case report.

\section{Disclosure Statement}

The authors declare that they have no conflicts of interest regarding the publication of this case report.

\section{Funding Sources}

This research received no specific grant from any funding agency in the public, commercial, or not-for-profit sectors.

\section{Author Contributions}

N.K. and N.S. conceived the work. D.K. and Y.H. supervised the work. N.K. and N.S. drafted the manuscript, and all authors contributed substantially to its revision. N.S. take responsibility for the paper as a whole. All authors read and approved the final manuscript.

\section{References}

1 Rogers PJ, Richardson NJ, Elliman NA. Overnight caffeine abstinence and negative reinforcement of preference for caffeine-containing drinks. Psychopharmacology (Berl). 1995 Aug;120(4):457-62.

2 Kamijo Y, Takai M, Fujita Y, Usui K. A Retrospective Study on the Epidemiological and Clinical Features of Emergency Patients with Large or Massive Consumption of Caffeinated Supplements or Energy Drinks in Japan. Intern Med. 2018 Aug;57(15):2141-6.

3 Willson C. The clinical toxicology of caffeine: A review and case study. Toxicol Rep. 2018 Nov;5:1140-52.

4 Wrenn KD, Oschner I. Rhabdomyolysis induced by a caffeine overdose. Ann Emerg Med. 1989 Jan;18(1):947. 
5 Campana C, Griffin PL, Simon EL. Caffeine overdose resulting in severe rhabdomyolysis and acute renal failure. The American journal of emergency medicine. 2014;32(1):111 e3-4. https://doi.org/10.1016/j.ajem.2013.08.042.

6 Jabbar SB, Hanly MG. Fatal caffeine overdose: a case report and review of literature. Am J Forensic Med Pathol. 2013 Dec;34(4):321-4.

7 Chen CY, Lin YR, Zhao LL, Yang WC, Chang YJ, Wu HP. Clinical factors in predicting acute renal failure caused by rhabdomyolysis in the ED. Am J Emerg Med. 2013 Jul;31(7):1062-6.

8 Bosch X, Poch E, Grau JM. Rhabdomyolysis and acute kidney injury. N Engl J Med. 2009 Jul;361(1):62-72.

9 Brown CV, Rhee P, Chan L, Evans K, Demetriades D, Velmahos GC. Preventing renal failure in patients with rhabdomyolysis: do bicarbonate and mannitol make a difference? J Trauma. 2004 Jun;56(6):1191-6.

10 Brochard L, Abroug F, Brenner M, Broccard AF, Danner RL, Ferrer M, et al.; ATS/ERS/ESICM/SCCM/SRLF Ad Hoc Committee on Acute Renal Failure. An Official ATS/ERS/ESICM/SCCM/SRLF Statement: Prevention and Management of Acute Renal Failure in the ICU Patient: an international consensus conference in intensive care medicine. Am J Respir Crit Care Med. 2010 May;181(10):1128-55.

11 Bolignano D, Coppolino G, Barillà A, Campo S, Criseo M, Tripodo D, et al. Caffeine and the kidney: what evidence right now? J Ren Nutr. 2007 Jul;17(4):225-34.

12 Zarich S, Fang LS, Diamond JR. Fractional excretion of sodium. Exceptions to its diagnostic value. Arch Intern Med. 1985 Jan;145(1):108-12.

13 Levey AS, James MT. Acute Kidney Injury. Ann Intern Med. 2017 Nov;167(9):ITC66-80.

14 Choudhury D, Ahmed Z. Drug-associated renal dysfunction and injury. Nat Clin Pract Nephrol. 2006 Feb;2(2):80-91.

15 Rossert J. Drug-induced acute interstitial nephritis. Kidney Int. 2001 Aug;60(2):804-17.

16 Cruz DN, Perazella MA. Drug-induced acute tubulointerstitial nephritis: the clinical spectrum. Hosp Pract (1995). 1998;33(2):151-64. https://doi.org/10.1080/21548331.1998.11443642.

17 Kamal F, Snook L, Saikumar JH. Rhabdomyolysis-Associated Acute Kidney Injury With Normal Creatine Phosphokinase. Am J Med Sci. 2018 Jan;355(1):84-7.

18 Kato H, Fujigaki Y, Inoue R, Asakawa S, Shin S, Shima T, et al. Therapeutic dose of acetaminophen as a possible risk factor for acute kidney injury: learning from two healthy young adult cases. Intern Med. 2014;53(14):1531-4.

19 Kher K, Makker S. Acute renal failure due to acetaminophen ingestion without concurrent hepatotoxicity. Am J Med. 1987 Jun;82(6):1280-1.

20 Bennett S, Hoffman N, Monga M. Ephedrine- and guaifenesin-induced nephrolithiasis. J Altern Complement Med. 2004 Dec;10(6):967-9.

21 Small E, Sandefur BJ. Acute renal failure after ingestion of guaifenesin and dextromethorphan. J Emerg Med. 2014 Jul;47(1):26-9. 